\title{
Initial and Post-Initial Code Acquisition in the Noncoherent Multiple-Input/Multiple-Output-Aided DS-CDMA Downlink
}

\author{
Seung Hwan Won, Member, IEEE, and Lajos Hanzo, Fellow, IEEE
}

\begin{abstract}
In this paper, we investigate the issues of both initial and post-initial acquisition schemes in the multiple-input/ multiple-output (MIMO)-aided direct-sequence code-division multiple-access (DS-CDMA) downlink when communicating over spatially uncorrelated Rayleigh channels. The associated mean acquisition time (MAT) performance trends are characterized as a function of the number of MIMO elements. Furthermore, we characterize both the initial and post-initial acquisition performance as a function of the relevant system parameters. Our findings suggest that increasing the number of transmit antennas in a MIMO-aided CDMA system results in combining the lowenergy noise-contaminated signals of the transmit antennas, which ultimately increases the MAT by an order of magnitude when the signal-to-interference-plus-noise ratio (SINR) is relatively low, regardless of whether single- or multipath scenarios are considered. This phenomenon has a detrimental effect on the performance of Rake-receiver-based synchronization schemes when the perfectly synchronized system is capable of attaining its target bit-error-rate performance at reduced SINR values, as a benefit of employing multiple transmit antennas. Based on our analysis justified by information-theoretic considerations, our acquisition design guidelines are applicable to diverse noncoherent (NC) MIMOassisted scenarios.
\end{abstract}

Index Terms-Initial acquisition, noncoherent (NC) multipleinput/multiple-output (MIMO) system, post-initial acquisition, serial search.

\section{INTRODUCTION}

A VARIETY OF schemes employing multiple antennas in the downlink (DL) of wireless systems constitutes an attractive technique of reducing the detrimental effects of time-variant multipath fading environments [1]. In intercell synchronous code-division multiple-access (CDMA) systems, the mobile station's (MS) receiver must be capable of perfectly synchronously aligning a locally generated pseudonoise (PN) code with the received multiuser signals containing the desired user's PN sequence [2], [3]. The novel technique of differential acquisition of $m$-sequences using recursive soft sequential estimation was proposed in [4], whereas a range of further re-

Manuscript received November 29, 2006; revised November 7, 2007 and January 11, 2008. First published October 31, 2008; current version published May 11, 2009. This work was supported in part by the Ministry of Information and Communication, Republic of Korea, by the Engineering and Physical Sciences Research Council, U.K., and by the European Union. The review of this paper was coordinated by Dr. K. T. Wong.

The authors are with the School of Electronics and Computer Science, University of Southampton, Southampton SO17 1BJ, U.K. (e-mail: lh@ecs.soton.ac.uk).

Digital Object Identifier 10.1109/TVT.2008.2007461 cent advances in multiple-input/multiple-output (MIMO)-aided acquisition has been disseminated in [5]-[7].

Our investigations in this paper consider the initial synchronization stage of CDMA systems, aiming for the acquisition of the coarse timing of the signals received in the DL. Therefore, our objective is to minimize the mean acquisition time (MAT), which is proportional both to the correct detection probability $P_{D}$ and to the time required by the acquisition scheme to notice after the elapse of the code-phase verification period that a false-locking event occurred and, hence, to return to the search mode. The uncertainty region in the DL corresponds to the entire period of the PN sequence, which tends to be quite wide, for instance, $\left(2^{15}-1\right)$ chip intervals in the DL of the intercell synchronous CDMA system [2], [8]. Therefore, the MAT is minimized in the context of serial search techniques by achieving the best possible $P_{D}$ while maintaining as low a value of the false-alarm probability $P_{F}$ and false-locking penalty as possible. However, most of these acquisition schemes have been designed for single-transmit/single-receive-antenna-aided systems, with the aim of optimizing the achievable MAT performance. Furthermore, there is a paucity of code-acquisition techniques designed for optimum post-initial acquisition performance [9], [10], where the term "post-initial acquisition" [9] refers to identifying the timing instants of the affordablecomplexity-dependent number of delayed received signal paths, which will be combined by the Rake receiver.

The substantial appeal of MIMOs is that their capacity linearly increases with the signal-to-interference-plus-noise ratio (SINR), as opposed to the more modest logarithmic increase of the classic Shannon-Hartley law, which may simply be exemplified by assigning the increased transmit power to an additional antenna and, hence, linearly increasing the throughput [11]. Ironically, when considering a fixed total power, but increasing the number of transmitter elements, the "per-element power" is reduced, which potentially results in a degraded acquisition performance, even if the associated degradation is partially compensated by increasing the number of receiver elements. This initial acquisition challenge has not been detailed in journal papers, although there are thousands of papers on showing the performance improvements of diverse MIMOs in perfect initial synchronization scenarios. Against this background, in this treatise, we investigate serial-search-based noncoherent (NC) code acquisition schemes designed for MIMO-aided systems to analyze the characteristics of both initial and post-initial acquisition schemes. More explicitly, we quantify both $P_{D}$ and $P_{F}$ as a function of both the SINR per chip, as well as that of the 
number of MIMO elements. Furthermore, we characterize the attainable MAT-versus- $\left(E_{c} / I_{0}\right)$ performance parameterized by the number of MIMO elements. Finally, based on our results justified by information theoretic considerations, we provide acquisition design guidelines applicable to diverse NC MIMOaided scenarios.

This paper is organized as follows: Section II describes the system investigated, followed by the $P_{D}$ and $P_{F}$ analysis of NC code acquisition schemes in the context of spatially uncorrelated Rayleigh channels, whereas the MAT analysis of both initial and post-initial acquisition is illustrated in Section III. In Section IV, our numerical MAT results are discussed, whereas our conclusions are offered in Section V.

\section{Correct Detection AND FALSE-ALARM PROBABILITY}

We assume that a finite-length tapped-delay line channel model generates $L$ Rayleigh-faded multipath signals, each arriving with a time delay $\tau_{l}$ having a tap spacing of one chip duration [3], [12], where $l=L$ is the number of multipath components. It is also assumed that Rayleigh fading is sufficiently slow for the faded envelope to remain constant over the duration of $\tau_{D}=N \cdot T_{c}$, but fast enough so that the consecutive $\tau_{D}$-duration segments may be considered essentially independently faded, as in [2], [13], and [14], where $\tau_{D}$ indicates the integral dwell time, $N$ represents the number of chips accumulated over the duration of $\tau_{D}$, and $T_{c}$ is the chip duration. Furthermore, the Neyman-Pearson criterion [2], [15] is adopted, which leads to a constant false-alarm rate. This is because as a benefit of normalizing the received signal by the background noise variance estimate, the fading channel's attenuation no longer affects the outcome of a test, whether the desired signal is present or absent. The resultant scenario and the related test become reminiscent of an additive white Gaussian noise (AWGN) scenario. Consequently, in line with the findings of [2], [13], and [14], the mobile channel only affects the $P_{D}$ performance. The spacing of both the multiple transmit elements at the BS and the multiple receive elements at the MS are assumed to be $10 \lambda$ and $0.5 \lambda$, respectively [16]. Accordingly, it is assumed that there is no spatial correlation among the DL signals emanating from the $P$ transmit antennas. Finally, we note that the analysis of our code acquisition schemes is valid for both spatial division multiplexing [17] and space-time coding [18] MIMOs.

The signal received over the multipath Rayleigh fading channel at each receive antenna of the MIMO-aided direct-sequence CDMA (DS-CDMA) DL may be expressed as [19]

$$
\begin{aligned}
& r_{n}(t)=\sum_{l=1}^{L} \sum_{m=1}^{P}\left[\alpha_{(l, m, n)} \sqrt{\frac{E_{c}}{P T_{c}}} c\left(t+d T_{c}+\tau_{l}\right)\right. \\
& \left.\cdot W_{m}\left(t+d T_{c}+\tau_{l}\right) \exp \left(2 \pi f t+\phi_{(l, m, n)}\right)+I_{(l, m, n)}(t)\right]
\end{aligned}
$$

where $m=P$ is the number of transmit antennas, $n=R$ is the number of receive antennas, $\alpha_{(l, m, n)}$ represents the complexvalued envelope of the $(l, m, n)$ th signal path obeying the Rayleigh distribution, $E_{c}$ denotes the pilot signal energy per
PN code chip, $c(t)$ represents a common PN sequence having a cell-specific code-phase offset, $d$ is the code-phase offset with respect to the phase of the local code, $W_{m}(t)$ identifies the specific Walsh code assigned to the $m$ th transmit antenna, $f$ is the carrier frequency, and finally, $\phi$ is the carrier phase of a specific user's modulator. Furthermore, $I_{(l, m, n)}(t)$ is the complex-valued AWGN having a double-sided power spectral density of $I_{0}$ at the $(l, m, n)$ th path. Here, the total allocated power is equally shared by the $P$ transmit antennas. Fig. 1 depicts the block diagram of the $\mathrm{NC}$ receiver designed for our code acquisition scheme using MIMO elements, which generates a decision variable by accumulating $(P \cdot R)$ independently faded signals observed over a time interval to improve the $P_{D}$ in the mobile channel imposing both fading and poor SINR conditions. In Fig. 1, the numbers 1 to $R$ surrounded by a circle situated in the upper right-hand corner of the largest box represent a specific receiver branch for each receive antenna. To simplify the receiver's structure, we omitted the front-end downconverter, the chip-matched filter, as well as the sampler and descrambler of the PN code. Further details on the related schemes may be found in [19].

To derive the probability density function (pdf) of the decision variable, let us now consider the effects of both the timing errors $\tau$ and those of the total frequency mismatch $\Delta f_{t}$ on the received signal. The timing errors are imposed by both the delay of the DL signal received from a mobile channel and the sampling inaccuracy caused by having a finite search step size of $\Delta=T_{c} / 2$. The total frequency mismatch is the sum of the clock-drift-induced frequency mismatch $\Delta f_{m}$ between the base station's (BS) transmitter and the MS's receiver, as well as of the effect of the Doppler shift $\Delta f_{d}$. If we take into account a strictly bandlimited transmit filter [2], the autocorrelation function (ACF) of the timing error $R(\tau)$ is expressed as ${ }^{1}$

$$
R(\tau)=\frac{\sin \left(\pi \tau / T_{c}\right)}{\left(\pi \tau / T_{c}\right)} \equiv \operatorname{sinc}\left(\tau / \mathrm{T}_{\mathrm{c}}\right)
$$

where $\tau$ is the arbitrary timing error. Due to the squaring operation in the NC receiver of Fig. 1, the square of $R(\tau)$ is considered to be the ACF of the timing errors. Similarly, the DL signal energy reduction, which is expressed as a function of the frequency mismatch after the same squaring operation $(\cdot)^{2}$ of Fig. 1, becomes [2]

$$
D\left(\Delta f_{t}\right) \approx \operatorname{sinc}^{2}\left(\mathrm{~N} \Delta \mathrm{f}_{\mathrm{t}} \mathrm{T}_{\mathrm{c}}\right) .
$$

\footnotetext{
${ }^{1}$ The timing error effects imposed are highly dependent on the type of the linear waveform-shaping filter transfer function used, rather than on the type of the spreading codes considered, since without shaping, they all exhibit rectangular chips. In our scenario, we opted for a strictly bandlimited frequencydomain chip-shaping filter transfer function, which is expressed as $H(f)=$ $(1 / \sqrt{W})[u(f+(W / 2))-u(f-(W / 2))]$, where $u(\cdot)$ denotes the unit step function, $f$ is the frequency in $H(f)$, and $W$ represents the total DS-spread bandwidth [2]. The resultant impulse response $h(t)$ is given by $h(t)=$ $\int_{-W / 2}^{W / 2} H(f) \exp (2 \pi i f t) d f=\sqrt{W} \sin (\pi W t) /(\pi W t)$, where $W \equiv 1 / T_{c}$. Therefore, without loss of generality, when using the squaring operation shown in Fig. 1, we have $X_{n}=+1$, where $n=1,2, \ldots, N$, and $X_{n}$ represents a squared chip value at the $n$th chip position, which effectively eliminates the influence of the polarity on the chip waveform. When we define the ACF $R(\tau)$ as $\int_{-\infty}^{\infty}|H(f)|^{2} \cos 2 \pi f \tau d f$, based on our assumption of the FD chipshaping filter, we have (2).
} 


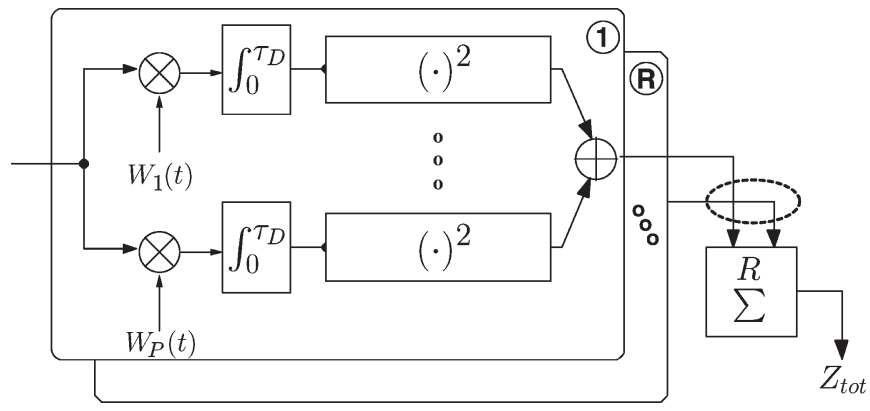

Fig. 1. Receiver structure of an $\mathrm{NC}$ code-acquisition system employing $R$ receive antennas. Its basic operation is identical for both the initial and postinitial acquisition schemes, except for using different coherent summation intervals necessitated by the different frequency mismatch of the two schemes.

Hence, the square of the mean values accumulated over $\tau_{D}$ is given by

$$
(\bar{N})^{2}=N^{2}\left(\frac{E_{c}}{P}\right) \operatorname{sinc}^{2}\left(\frac{\tau}{\mathrm{T}_{\mathrm{c}}}\right) \operatorname{sinc}^{2}\left(\mathrm{~N} \Delta \mathrm{f}_{\mathrm{t}} \mathrm{T}_{\mathrm{c}}\right) .
$$

As a result of the preceding formulation, the effects of both timing errors and frequency mismatches are encapsulated by the definition of the squared mean $(\bar{N})^{2}$ formulated in (4). More explicitly, this means that the effects of both of these detrimental factors are directly involved in the energy value measured at the output of the $(\cdot)^{2}$ block of Fig. 1. Accordingly, $\left(E_{c} / I_{0}\right)^{\prime}$ is defined as $\left(E_{c} / I_{0}\right) \cdot \operatorname{sinc}^{2}\left(\tau / \mathrm{T}_{\mathrm{c}}\right) \cdot \operatorname{sinc}^{2}\left(\mathrm{~N} \Delta \mathrm{f}_{\mathrm{t}} \mathrm{T}_{\mathrm{c}}\right)$, and this definition will be used in the definition of the noncentrality parameter. By employing the procedures proposed in [19] in the context of the receiver structure of Fig. 1, the final decision variable of the $l$ th path may be written as

$$
\begin{aligned}
Z_{\mathrm{tot}} & =\sum_{m=1}^{P} \sum_{n=1}^{R} Z_{k(l, m, n)} \\
& =\sum_{m=1}^{P} \sum_{n=1}^{R}\left\|\frac{1}{\sqrt{2}}\left(\sqrt{\frac{4 E_{c}}{N I_{0} P}} S_{k(l, m, n)}+I_{k(l, m, n)}\right)\right\|^{2}
\end{aligned}
$$

where $k$ denotes the $k$ th chip's sampling instant; $Z_{k(l, m, n)}$ is a decision variable of the $(m, n)$ th path, which constitutes an element of the final decision variable $Z_{\text {tot }}$; and $S_{k(l, m, n)}=$ $\left(1 / T_{c}\right) \int_{0}^{N T_{c}} c(t) c\left(t+d T_{c}+k T_{c}\right) \cdot \exp \left(j 2 \pi N \Delta f_{t}\right) d t$. If the PN codes have ideal ACFs, where the ACF has identical sidelobes to those of maximum-length shift-register sequences [2], $S_{k(l, m, n)}$ can be expressed as $N \cdot \exp \left(j 2 \pi N \Delta f_{t}\right)$ for the signal being present. On the other hand, in the case of the signal being absent, it can be shown to be $-1 \cdot \exp \left(j 2 \pi N \Delta f_{t}\right)$. Therefore, $S_{k(l, m, n)}$ becomes deterministic [19], whereas $I_{k(l, m, n)}$ is the complex-valued AWGN having zero means and variances of $\sigma^{2}=2$ for both their real and imaginary parts. Furthermore, $\|\cdot\|^{2}$ represents the Eucledian norm of the complex-valued argument in (5), and the factor of $1 / \sqrt{2}$ is employed to normalize according to the noise variance. It is worth noting that the outputs of the squaring operation invoked for both the in-phase and the quadrature branch of $Z_{k(l, m, n)}$ in Fig. 1 are modeled as the square of the Gaussian random variable, respectively.
Accordingly, the decision variable $Z_{k(l, m, n)}$ of each path obeys a noncentral chi-square pdf with two degrees of freedom [3] and having a noncentrality parameter of $\lambda_{x}$, which is either $(2 N / P)\left(E_{c} / I_{0}\right)$ for the hypothesis of the desired signal being present $\left(H_{x}, x=1\right)$ or $(2 / N P)\left(E_{c} / I_{0}\right)$ for it being absent $\left(H_{x}, x=0\right)$ [19]. This pdf is given by [12]

$$
f_{Z_{k(l, m, n)}}\left(z \mid H_{x}\right)=\frac{1}{2} \cdot \exp \left[-\frac{\left(z+\lambda_{x}\right)}{2}\right] \cdot \mathcal{I}_{0}\left(\sqrt{z \cdot \lambda_{x}}\right)
$$

where $z \geq 0, x=0$ or 1 , and $\mathcal{I}_{0}(\cdot)$ is the zeroth-order modified Bessel function. Now, our aim is that of expressing the pdf of a desired user's signal at the output of the acquisition scheme conditioned on the presence of the desired signal in $f_{Z_{\text {tot }}}\left(z \mid H_{x}\right)$ derived for transmission over a spatially uncorrelated Rayleigh channel. In this scenario, $E_{c}$ is multiplied by the square of the Rayleigh-distributed fading amplitude $\beta$, which exhibits a chi-square distribution having two degrees of freedom, and it is hence expressed as $f(\beta)=e^{-\beta / \sigma^{2}} / \sigma^{2}$, where $\sigma^{2}$ is the variance of the constituent Gaussian distribution. Then, the average pilot signal energy $\overline{E_{c}}$ per PN code chip can be expressed as $\overline{E_{c}}=\bar{\beta} E_{c}=\sigma^{2} E_{c}$ [2]. Therefore, first, the pdf $f_{Z_{k(l, m, n)}}\left(z \mid H_{x}, \beta\right)$ corresponding to $\beta$ conditioned on the hypothesis of the desired signal being transmitted over an AWGN channel having this specific SINR is weighted by the probability of occurrence $f(\beta)$ of encountering $\beta$, as quantified by the pdf. The resultant product is then averaged over its legitimate range of $-\infty \sim \infty$, yielding

$$
\begin{aligned}
f_{Z_{k(l, m, n)}}\left(z \mid H_{x}\right)= & \int_{-\infty}^{\infty} f(\beta) \cdot f_{Z_{k(l, m, n}}\left(z \mid H_{x}, \beta\right) d \beta \\
= & \int_{0}^{\infty}\left(\frac{e^{-\beta / \sigma^{2}}}{\sigma^{2}}\right) \cdot \frac{\exp \left[-\left(z+\beta \lambda_{x}\right) / 2\right]}{2} \\
& \cdot \mathcal{I}_{0}\left(\sqrt{\beta \lambda_{x} z}\right) d \beta \\
= & \frac{\exp \left[-z /\left(2+\lambda_{x} \sigma^{2}\right)\right]}{\left(2+\lambda_{x} \sigma^{2}\right)} \\
\equiv & \frac{\exp \left[-z /\left(2+\overline{\lambda_{x}}\right)\right]}{\left(2+\overline{\lambda_{x}}\right)}
\end{aligned}
$$

where the effects of both timing errors and frequency mismatches are encapsulated by the definition of $\left(\overline{E_{c} / I_{0}}\right)^{\prime}$, and the corresponding noncentrality parameter $\overline{\lambda_{x}} \equiv \lambda_{x} \sigma^{2}$ is either $(2 N / P)\left(\overline{E_{c}} / I_{0}\right)^{\prime}$, when the desired signal is deemed to be present $\left(H_{x}, x=1\right)$, or $(2 / N P)\left(\overline{E_{c}} / I_{0}\right)^{\prime}$, when it is deemed to be absent $\left(H_{x}, x=0\right)$. We also define $\mu_{x}=\left(2+\overline{\lambda_{x}}\right)$, which physically represents a new biased noncentrality parameter. Further details on the related calculations can be found in [2] and [19]. Finally, we arrive at the pdf of $Z_{k(l, m, n)}$ conditioned on the presence of the desired signal in the form of

$$
f_{Z_{k(l, m, n)}}\left(z \mid H_{x}\right)=\frac{1}{\mu_{x}} e^{-z / \mu_{x}} .
$$

Since the decision variable $Z_{\text {tot }}$ is constituted by the sum of $(P \cdot R)$ number of independent variables, which has a pdf given 
by (11), we can determine the Laplace transform of each by raising them to the $(P \cdot R)$ th power and then carrying out the inverse transform to generate the desired pdf [2], leading to

$$
f_{Z_{\mathrm{tot}}}\left(z \mid H_{x}\right)=\frac{z^{(P R-1)} e^{-z / \mu_{x}}}{\Gamma(P R) \cdot \mu_{x}^{P R}}
$$

where $\Gamma(\cdot)$ is the Gamma function. Finally, the probability of correct detection or false alarm, corresponding to $\xi=D$ or $F$, respectively, is obtained as

$$
\begin{aligned}
\left.P\right|_{\xi=D o r F} & =\left.\int_{\theta}^{\infty} f_{Z_{\mathrm{tot}}}\left(z \mid H_{x}\right) d z\right|_{x=1 \text { or } 0} \\
& =\exp \left(-\frac{\theta}{\mu_{x}}\right) \cdot \sum_{k=0}^{P R-1} \frac{\left(\theta / \mu_{x}\right)^{k}}{k !}
\end{aligned}
$$

where $\theta$ is a threshold value.

\section{MAT ANALYSIS OF INITIAL AND POST-INITIAL ACQUISITION}

The classic serial search techniques designed for initial acquisition [2] have traditionally been employed in specific scenarios, where the uncertainty region (or search window width) is quite wide (i.e., $2^{15}-1$ ), and the length of the PN sequence in our system was assumed to be $\left(2^{15}-1\right) \cdot T_{c}$, as shown, for example, in the DL of the intercell synchronous CDMA2000 system [2], where the chip duration is $T_{c}=1 / 1.2288 \mu \mathrm{s}$. In the case of initial acquisition contrived for DS-CDMA, the main design goal is to acquire perfect timing of the first received signal path impinging at the receiver, since this timing information is used as that of the reference finger of the Rake receiver. By contrast, the post-initial acquisition procedure, which extracts the accurate timing positions of the remaining delayed paths and identifies the appropriate paths earmarked for processing by the maximum ratio combining scheme of the Rake receiver, has a major impact on the performance of the Rake receiver [10]. There are two main differences between the initial and post-initial acquisition procedures. First, once the first Rake finger is synchronized, the uncertainty region that has to be explored will be shrunk to $\pm U$ hypotheses surrounding the time instant, where the first received path was found, and $U$ represents the reduced uncertainty region to be explored after the initial acquisition [9]. This search window width is defined both by the dispersion of the multipath propagation environment encountered and by the appearance and disappearance of propagation paths [20]. Second, the post-initial acquisition procedure commences after automatic frequency control operation was activated for fine tracking, following the successful initial acquisition. Hence, the performance degradation imposed by the associated frequency mismatch is considerably reduced compared to that immediately after the initial acquisition. Accordingly, these two factors are taken into account in our forthcoming analysis.

In [2] and [8], explicit MAT formulas were provided for a single-antenna-aided serial-search-based code acquisition system. There is no distinction between single-antenna-aided and multiple-antenna-assisted schemes in terms of analyzing the MAT, except for deriving $P_{D}$ and $P_{F}$ based on the MIMO elements. We will commence our discourse by analyzing the MAT performance of both initial and post-initial acquisition schemes employing both single-dwell serial search (SDSS) and double-dwell serial search (DDSS). We assume that in each chip duration $T_{c}, \alpha$ number of correct timing hypotheses are tested, which are spaced by $T_{c} / \alpha$. Hence, the total uncertainty region is increased by a factor of $\alpha$. Moreover, as mentioned in Section II, when the $L$ multipath signals arrive with a time delay $\tau_{l}$ having a tap spacing of one chip duration, then the relative frequency of the signal being present is increased by $L$-fold. The required transfer functions [2], [8] are defined as follows: The entire successful detection function $H_{D}(z)$ encompasses all the branches of a state diagram [2], [8], which leads to successful detection. Furthermore, $H_{0}(z)$ indicates the absence of the desired user's signal at the output of the acquisition scheme, whereas $H_{M}(z)$ represents the overall miss probability of a search run carried out across the entire uncertainty region. Since all the nodes of the state diagram are assumed to be equally likely a priori, the resultant transfer function averaged over all the $(\nu-2 \alpha L)$ root nodes or starting nodes becomes [2], [8]

$$
\begin{aligned}
T(z) & =\frac{1}{(\nu-2 \alpha L)} \sum_{i=1}^{(\nu-2 \alpha L)} \frac{H_{0}^{i}(z) H_{D}(z)}{\left[1-H_{M}(z) H_{0}^{(\nu-2 \alpha L)}(z)\right]} \text { (15) } \\
& =\frac{H_{D}(z) H_{0}(z)\left[1-H_{0}^{(\nu-2 \alpha L)}(z)\right]}{(\nu-2 \alpha L)\left[1-H_{0}(z)\right]\left[1-H_{M}(z) H_{0}^{(\nu-2 \alpha L)}(z)\right]} .
\end{aligned}
$$

Equation (16) encompasses an average of all the information with regard to both the time instant and the probability of the $i$ th path in the resultant transfer function. By employing the differentiation of $\left.T(z)\right|_{z=1}$, it may be shown that the generalized expression derived for computing the MAT of the serial-searchbased code acquisition scheme is given by [2], [3], [8]

$$
\begin{aligned}
& E\left[T_{\mathrm{ACQ}}\right] \\
& =\frac{1}{H_{D}(1)}\left[H_{D}^{\prime}(1)+H_{M}^{\prime}(1)\right. \\
& \left.\quad+\left\{(\nu-2 \alpha L)\left[1-\frac{H_{D}(1)}{2}\right]+\frac{1}{2} H_{D}(1)\right\} H_{0}^{\prime}(1)\right] \cdot \tau_{D 1} \\
& \approx \frac{\left(1+H_{M}(1)\right) \cdot H_{0}^{\prime}(1)}{2 \cdot\left(1-H_{M}(1)\right)} \cdot\left(\nu \cdot \tau_{D 1}\right)
\end{aligned}
$$

where $\left.H_{x}^{\prime}(z)\right|_{x=D, M \text { or } 0}$ is a derivative of $\left.H_{x}(z)\right|_{x=D, M \text { or } 0, \nu}$ represents the total number of states to be searched, and $\tau_{D 1}$ denotes the first dwell time. The related processes are further detailed for SDSS in [2] and for DDSS in [8], [21], and [22]. The exact MAT formula can be simplified if $\nu$ is significantly higher than the number of $H_{D}$ states [3]. To simplify our 
TABLE I

MAXIMUM SINR DEGRADATION INFLICTED BY BOTH THE DOPPLER SHIFT AND A 1000-Hz FREQUENCY MISMATCH, IN CONJUNCTION WITH THE COHERENT INTEGRATION INTERVAL OF $N$ CHIP DURATIONS AT A CARRIER FREQUENCY OF $1.9 \mathrm{GH} z$

\begin{tabular}{|l|l|l|l|l|l|}
\hline $\mathrm{N}($ Chips $)$ & 64 & 128 & 256 & 384 & 512 \\
\hline Degradation $(\mathrm{dB})$ & 0.061 & 0.2449 & 0.9969 & 2.3144 & 4.3213 \\
\hline
\end{tabular}

TABLE II

MAXIMUM SINR DEGRADATION INFLICTED BY BOTH THE DOPPLER ShifT AND A 200-Hz FreQuenCy Mismatch, IN CONJUnCTION WITH THE COHERENT INTEGRATION INTERVAL OF $N$ CHIP DURATIONS AT A CARRIER FREQUENCY OF $1.9 \mathrm{GHz}$

\begin{tabular}{|l|l|l|l|l|l|l|}
\hline $\mathrm{N}$ (Chips) & 128 & 256 & 384 & 512 & 640 & 768 \\
\hline Degradation $(\mathrm{dB})$ & 0.032 & 0.128 & 0.289 & 0.5159 & 0.812 & 1.179 \\
\hline
\end{tabular}

numerical performance analysis, we adopted the approximation of the exact MAT expression proposed in [3]. Since each resolvable path contributes two hypotheses of the signal being present and because the average $P_{D}$ associated with these two hypotheses is the same, the overall miss probabilities of both the SDSS and DDSS schemes may be expressed as

$$
\begin{aligned}
& H_{M}(1)=\prod_{l=1}^{L} \prod_{\zeta=1}^{\alpha}\left(1-P_{D(l, \zeta)}\right)^{2} \\
& H_{M}(1)=\prod_{l=1}^{L} \prod_{\zeta=1}^{\alpha}\left[\left(1-P_{D 1(l, \zeta)}\right)+P_{D 1(l, \zeta)} \cdot\left(1-P_{D 2(l, \zeta)}\right)\right]^{2}
\end{aligned}
$$

respectively, where $P_{D(l, \zeta)}$ represents the correct detection probability of the SDSS scheme, and $\left.P_{D x(l, \zeta)}\right|_{x=1 \text { or } 2}$ are the correct detection probabilities of both the search and verification modes of the DDSS arrangements, respectively. Both $P_{D(l, \zeta)}$ and $P_{D x(l, \zeta)}$ are given by (14), provided that the condition of the signal being present is satisfied. The $H_{0}^{\prime}(1)$ values of the SDSS and DDSS schemes are expressed as

$$
\begin{aligned}
& H_{0}^{\prime}(1)=1+K \cdot P_{F} \\
& H_{0}^{\prime}(1)=1+m \cdot P_{F 1}+K \cdot P_{F 1} \cdot P_{F 2}
\end{aligned}
$$

respectively, where $K$ denotes the false-locking penalty factor, which is expressed in terms of the number of chip intervals required by an auxiliary device for recognizing that the codetracking loop is still unlocked; and $m$ represents the exponent of $z$ in the verification mode. Furthermore, $P_{F}$ is the false-alarm probability of the SDSS scheme, and $\left.P_{F x}\right|_{x=1 \text { or } 2}$ represent the false-alarm probability in both the search and verification modes of the DDSS scheme, respectively. Similarly, both $P_{F}$ and $P_{F_{x}}$ are given by (14), provided that the condition of the signal being absent is satisfied.

\section{Numerical System Performance Results}

In this section, we will characterize the MAT performance of the MIMO-aided DS-CDMA code-acquisition scheme of Fig. 1. In Tables 1 and II, we outlined the maximum SINR degradation imposed by both the Doppler shift and the frequency mismatch between the transmitter and receiver, in con-
TABLE III

Optimized Length of CoHEREnT Summation of Detector OutPuts INVOKED TO ANALYZE SDSS IN POST-INITIAL ACQUISITION

\begin{tabular}{|l|l|l|l|l|l|l|l|}
\hline Tx/Rx & P1R1 & P2R1 & P4R1 & Tx/Rx & P1R4 & P2R4 & P4R4 \\
\hline $\begin{array}{l}\text { Length } \\
\text { (Chips) }\end{array}$ & 512 & 512 & 640 & $\begin{array}{l}\text { Length } \\
\text { (Chips) }\end{array}$ & $\begin{array}{l}256 \\
(128)\end{array}$ & $\begin{array}{l}256 \\
(384)\end{array}$ & 384 \\
\hline
\end{tabular}

TABLE IV

Optimized Length of COHERENT Summation of Detector OutPuts INVOKED IN THE VERIFICATION MODE TO ANALYZE DDSS IN POST-INITIAL ACQUISITION

\begin{tabular}{|l|l|l|l|l|l|l|l|}
\hline Tx/Rx & P1R1 & P2R1 & P4R1 & Tx/Rx & P1R4 & P2R4 & P4R4 \\
\hline $\begin{array}{l}\text { Length } \\
\text { (Chips) }\end{array}$ & 384 & 640 & 768 & $\begin{array}{l}\text { Length } \\
\text { (Chips) }\end{array}$ & $\begin{array}{l}256 \\
(384)\end{array}$ & 384 & 512 \\
\hline
\end{tabular}

junction with the coherent integration interval of $\tau_{D}$ durations shown in Fig. 1 for both initial and post-initial acquisition. In the case of the initial acquisition scheme of Fig. 1, it was found to be sufficient to integrate the detector output shown in Fig. 1 over $N=128$ chips to analyze SDSS, whereas the number of chips over which the accumulator $\Sigma$ of Fig. 1 sums the $(\cdot)^{2}$ envelope detector's output in both the search and verification modes of DDSS is assumed to be 32 and 256 in the $R=1$ receive antenna scenario or 128 in the $R=4$ receive antennas scenario, respectively. By contrast, in the case of the postinitial acquisition scheme, the appropriate length of coherent summation of the detector output values invoked to analyze SDSS is given in Table III, whereas 64 is selected as the length of coherent summation in the search mode of DDSS. Finally, the appropriate intervals of the coherent summation used in the verification mode of DDSS are portrayed in Table IV. In Tables III and IV, the numbers shown in $(\cdot)$ can be used as an alternative. These beneficially chosen parameter values were calculated by using the results of Tables I and II based on (3), as well as taking into account the effects of the sampling inaccuracy, quantified on the basis of (14) and (16), and then finding the parameter values, which resulted in the best achievable MAT performance. The spreading factor of the Walsh code to be acquired was selected to be 128 . The frequency mismatch was assumed to be $1000 \mathrm{~Hz}$ for the initial acquisition phase [2] and $200 \mathrm{~Hz}$ for the post-initial acquisition phase [10], whereas the carrier frequency was $1.9 \mathrm{GHz}$. As the worst-case mobile speed, it is reasonable to postulate $160 \mathrm{~km} / \mathrm{h}$. We also assumed that the sampling inaccuracy caused by having a finite, rather than infinitesimally low, search step size of $\Delta=T_{c} / 2$ was $-0.91 \mathrm{~dB}$, which is a typical value for the search step size [2]. Indeed, when considering multiple hypothesis tests per chip, the effects of cell correlation become nonnegligible [23]. However, in the case of $\Delta=T_{c} / 2$, the effect of cell correlation becomes modest [23]; hence, it is reasonable to assume that two consecutive cells are uncorrelated. The total uncertainty regions of the initial and post-initial acquisition were assumed to entail 65534 and 124 hypotheses, respectively. In the spirit of [8], the false-locking penalty factor was assumed to be 1000 chip durations. Finally, both single- and multipath scenarios were considered. Three paths arriving with a relative time delay of one chip and having a magnitude difference of $3 \mathrm{~dB}$, respectively, were assumed to be present in a given search window. All the performance curves have been obtained at the 


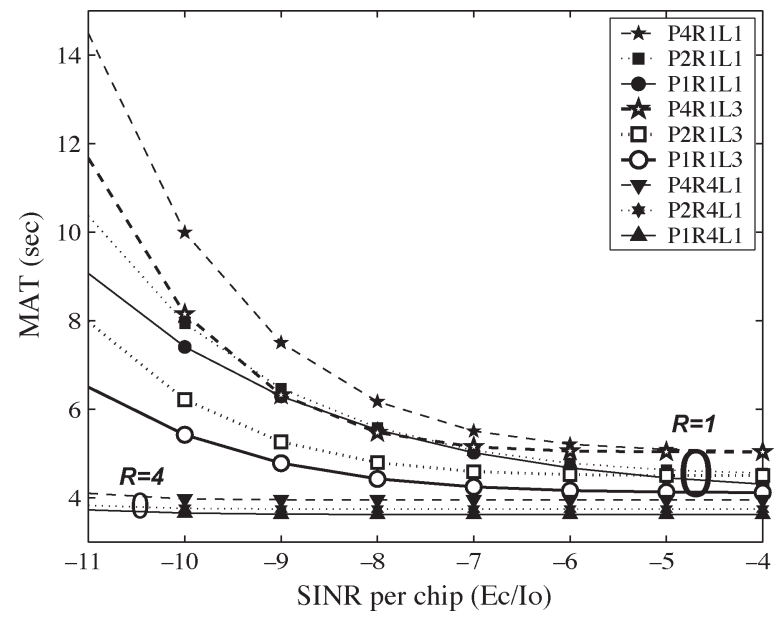

Fig. 2. MAT-versus-SINR-per-chip performance of the initial acquisition system for SDSS parameterized with both the number of transmit and receive antennas.

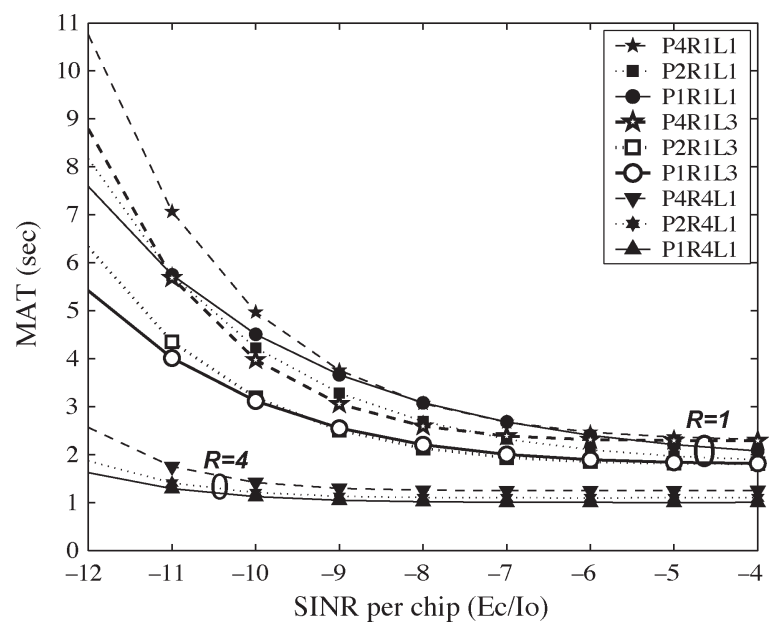

Fig. 3. MAT-versus-SINR-per-chip performance of the initial acquisition system for DDSS parameterized with both the number of transmit and receive antennas.

decision threshold of $E_{c} / I_{0}=-13 \mathrm{~dB}$ optimized for the initial acquisition scheme and at $E_{c} / I_{0}=-19 \mathrm{~dB}$ invoked for the post-initial acquisition scheme, respectively. The operational range of the post-initial acquisition scheme was assumed to be $6 \mathrm{~dB}$ lower than that of the initial acquisition arrangement because the signal power of the delayed paths is typically lower than that of the first received path.

Fig. 2 illustrates the achievable MAT-versus-SINR-per-chip performance for SDSS of the initial acquisition scheme as a function of the number of transmit antennas for $P=1,2$, and 4 and that of the number of receive antennas for $R=1$ and 4. In the results of Figs. 2-6, except for Fig. 4, the bold lines indicate the scenario of encountering three paths (denoted as L3 in Figs. 2-6, except for Fig. 4), whereas the thinner lines represent a single-path scenario (denoted as $L 1$ in Figs. 2-6, except for Fig. 4). Observe in Fig. 2 that somewhat surprisingly, as the number of transmit antennas is decreased, despite the potentially reduced transmit diversity gain, we experience an improved MAT performance for both the single- and multipath scenarios. Since the number of states when the signal is present

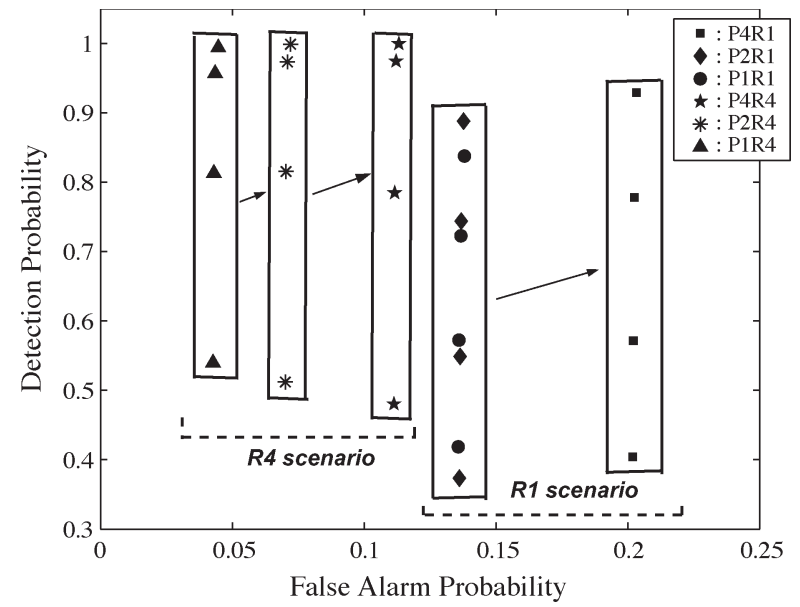

Fig. 4. Operating ranges in the search mode of the initial acquisition scheme to obtain the best possible MAT performance. (Top to bottom) Four vertically stacked points corresponding to $E_{c} / I_{o}=-4,-7,-10$, and $-13 \mathrm{~dB}$, respectively.

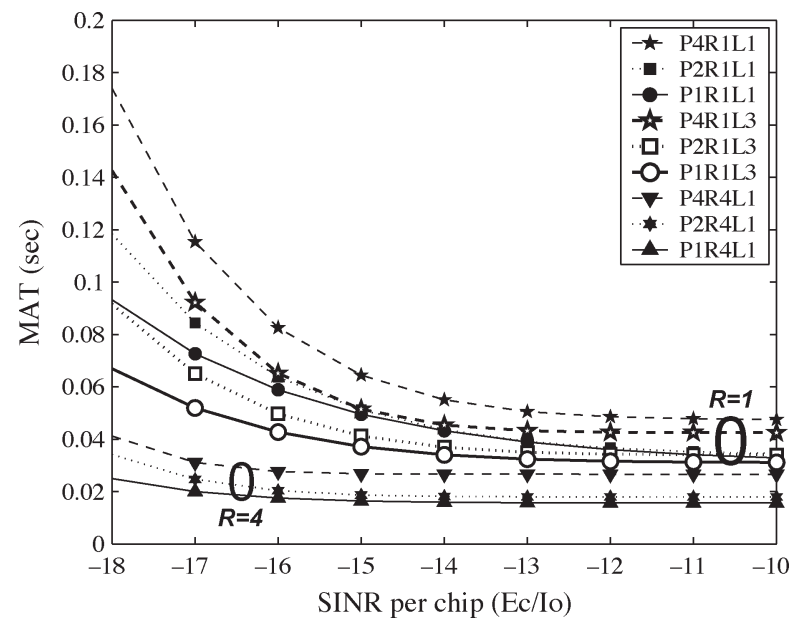

Fig. 5. MAT-versus-SINR-per-chip performance of the post-initial acquisition system for SDSS parameterized with both the number of transmit and receive antennas.

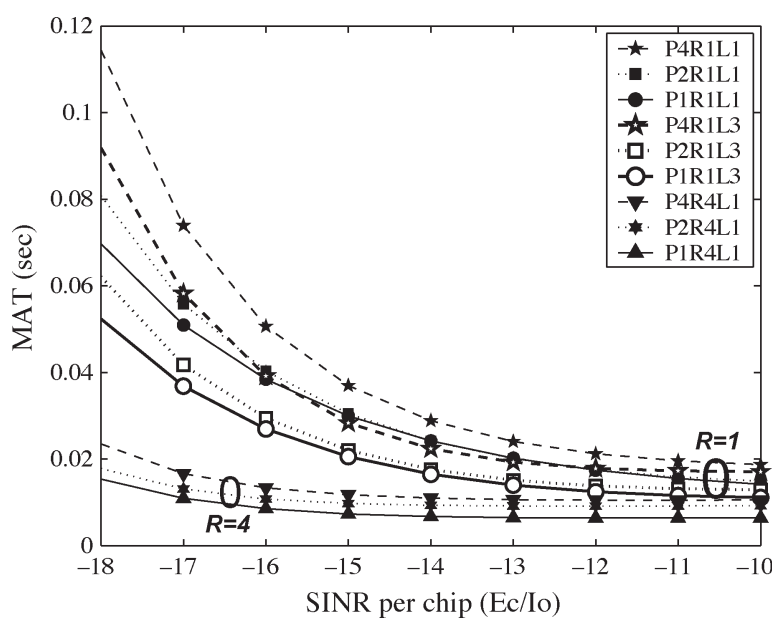

Fig. 6. MAT-versus-SINR-per-chip performance of the post-initial acquisition system for DDSS parameterized with both the number of transmit and receive antennas. 
was increased by a factor of $L=3$, the MAT performance of this scenario becomes better than that of the single-path scenario. In the case of $R=4$ receivers, the performance improvements due to having multiple paths become marginal, because the receive diversity gain is already sufficiently high for having a near-Gaussian MAT performance. Hence, the results of the multipath scenario of $R=4$ were omitted to avoid obfuscating details. For comparison, Fig. 3 characterizes the MAT-versusSINR-per-chip performance of DDSS for the initial acquisition arrangement as a function of the number of transmit antennas for $P=1,2$, and 4 and that of the number of receive antennas for $R=1$ and 4. Similarly to the conclusions of Fig. 2, as the number of transmit antennas is decreased, all the curves shown in Fig. 3 illustrate an improved MAT performance, except that a useful transmit diversity gain is experienced only for the case of " $P 2 R 1$," and even this gain was limited to the specific SINR range of -4 and $-11 \mathrm{~dB}$ in the single-path scenario. In the case of DDSS, the performance improvements obtained for the three-path scenario are less than those of SDSS. It is worth mentioning that although not explicitly shown in Figs. 2, 3, 5, and 6 to avoid obfuscating details, the operating range of $R=2$ receive antennas was found to be between that corresponding to the $R=1$ and $R=4$ receive antenna scenario. To further illustrate the aforementioned fact, in the case of "P2R1," the DDSS scheme exhibits a better MAT performance compared to the " $P 1 R 1$ " arrangement across the specific SINR range shown in Fig. 3. This is because in the case of DDSS, the reliable operational ranges in terms of both the correct detection and false-alarm probabilities are quite different from those of SDSS. More explicitly, the reliable operational range of SDSS associated with the best possible MAT performance is around a false-alarm probability of $10^{-4}$. On the other hand, the reliable operation of DDSS may be maintained at a false-alarm probability as high as 0.2 when the number of transmit antennas is increased from $P=1$ to $P=4$, in conjunction with $R=1$ receive antenna, as demonstrated in Fig. 4. Furthermore, in the case of $R=4$ receive antennas, similar trends are observed, although the region of the reliable DDSS operation is shifted to the left with respect to the case of a single receive antenna, as shown in Fig. 4. It is worth mentioning that the operating range of $R=2$ receive antennas is in between that corresponding to $R=1$ and $R=4$ receive antennas; to avoid obfuscating points in the figure, the $R=2$ scenario was omitted. Accordingly, while the reliable operational range of SDSS is around a falsealarm probability of $10^{-4}$, that of DDSS in the search mode more widely varies, namely, across the range spanning from 0.04 to just over 0.2 , depending on the specific number of MIMO elements. This manifests itself also in terms of having detection threshold values in the search mode of DDSS, which are substantially lower than those of SDSS, when optimized to attain the best possible MAT performance. This clearly implies that DDSS benefits from a significantly higher diversity gain than SDSS. The performance degradation imposed by employing multiple antennas becomes more drastic, as the number of transmit antennas is increased for both the SDSS and DDSS schemes in the initial acquisition scenario. Furthermore, the associated MAT performance discrepancy between the SDSS and DDSS schemes becomes more drastic. In the case of em- ploying both multiple transmit and multiple receive antennas, similar trends are observable, although using four receive antennas has the potential of mitigating the associated acquisition performance degradation imposed by the low per-branch $E_{c} / I_{0}$ values associated with the employment of multiple transmitters.

Figs. 5 and 6 characterize the achievable MAT-versus-SINRper-chip performance of post-initial acquisition. The results are parameterized by both the number of transmit antennas for $P=1,2$, and 4 and by the number of receive antennas for $R=1$ and 4 for both the SDSS (Fig. 5) and DDSS schemes (Fig. 6), respectively. Although the optimized coherent summation intervals determined to obtain the best possible MAT performance are quite different, as the number of transmit antennas is decreased, all the curves shown in Figs. 5 and 6 indicate an improved MAT performance, as we observed in the case of initial acquisition in Figs. 2 and 3. This trend explicitly indicates that the DDSS scheme also degrades the achievable MAT performance as a consequence of the low per-antenna power imposed by employing multiple transmit antennas to attain a transmit diversity gain. However, the MAT performance degradation imposed is less severe than that of the SDSS scheme. Moreover, the performance improvements of the initial acquisition scheme recorded for SDSS in Fig. 2 as a benefit of having multiple paths is more significant than those of the post-initial acquisition arrangement shown for SDSS in Fig. 5. The initial acquisition scheme having multiple received paths contributes to the attainable performance improvements. On the other hand, in the case of DDSS, the performance improvements obtained for the three-path scenario are lower than those of SDSS in both the initial and postinitial acquisition scenarios. The performance improvements due to having multiple paths become the lowest for the postinitial acquisition arrangement using DDSS in Fig. 6. To further interpret all the aforementioned results, a low level of perbranch received signal strength would lead to low acquisition performance, despite achieving a high transmit diversity gain. In other words, a high transmit diversity order effectively results in an acquisition performance loss, as a consequence of the insufficiently high signal strength per transmit antenna branch.

Based on the aforementioned analysis, the detrimental factors governing the best attainable MAT performance are classified according to the following: 1) the value of the clock-drift-induced frequency mismatch and 2) the number of transmit antennas. By contrast, the beneficial factors are categorized according to the following four classes: 1) the length of coherent summation, 2) the number of receive antennas, 3 ) the number of multipath components, and 4) the number of dwell intervals. The main reasons for the aforementioned performance trends may further be justified by a number of information theoretic considerations applied to the NC MIMOaided scenarios, as follows [24]-[26].

Wireless systems employing MIMOs exhibit a high capacity, provided that the channel is known to the receiver [27]. By contrast, an NC MIMO-aided scheme that does not rely on any channel knowledge has a lower capacity [24]-[26]. However, it was argued in [25] that there is no reason for using more than $T_{S} / 2$ number of transmit antennas, where based on the results of Tables I and II, $T_{S}=2$ was the number of symbols over 
which integration is carried out, because the number of degrees of freedom increases with $T_{S} / 2$, but only until $P$ approaches $T_{S} / 2$ [25], where $P$ is the number of transmit antennas. Furthermore, at low SINRs, the mutual information between the transmitter and receiver is maximized by using a single transmit antenna, because the mutual information bounds were shown to be decreasing functions of $P$ [26]. This implies that using multiple transmit antennas provides no MAT performance gain in the low SINR region; it rather leads to a MAT performance degradation. Finally, in the medium SINR range, a maximum of $T_{S}$ transmit antennas is worth employing to achieve a MAT performance gain because the capacity achieved for $P>T_{S}$ is the same as that achieved for $P=T_{S}$ [24]. This indicates that $P=2$ transmit antennas are capable of achieving an improved MAT performance in the SINR region of -4 and $-11 \mathrm{~dB}$ in the single-path scenario of Fig. 3. Finally, when considering the design of MIMO-aided code-acquisition schemes, the following guidelines may be inferred.

1) Using multiple transmit antennas typically leads to a MAT performance degradation, except for the " $P 2 R 1$ " scenario encountering a single-path environment. Using a relatively low number of chips over which integration or accumulation is carried out imposes further limits on the attainable benefits of MIMOs. Furthermore, in the multipath scenarios considered, all the results fail to show a transmit diversity gain. Therefore, employing a single transmit antenna might be recommended for maximizing the achievable performance of both the initial and postinitial acquisition schemes investigated. Furthermore, using a sufficiently high number of postdetection integration (PDI) stages is also beneficial for minimizing the MAT. ${ }^{2}$

2) Using multiple receive antennas increases the achievable receiver diversity gain and has the potential of compensating for the MAT degradation imposed by the low perbranch power of multiple transmitters.

3) Acquiring the exact timing information of the received paths, specifically designed preambles, such as that of the primary synchronization channel of wideband CDMA [28] combined with time-switched transmit diversity, might be recommended, which is capable of achieving a diversity gain with the aid of a single transmit antenna [25], [26]. In practical scenarios, the received path timing differences of the signals arriving from multiple transmit antennas might be distributed within a fraction of a chip duration [29], although they may vary due to the timevariant propagation delay; hence, using multiple transmit antennas may further degrade the performance. In addition to initial acquisition, the classic pilot channel may also be used for carrier frequency error correction and channel estimation.

\footnotetext{
${ }^{2}$ The underlying philosophy of PDI is that a decision variable is generated by accumulating $T$ consecutive $N$-spaced signal samples observed over multiple $N$-spaced time intervals to improve the $P_{D}$ in the mobile channel imposing both fading and poor SINR conditions. This specific number must be determined by satisfying a pair of targeted $P_{D}$ and $P_{F}$ values to minimize the MAT.
}

\section{CONCLUSION}

In this paper, we have analyzed the MIMO-aided diversity effects on the performance of both initial and post-initial acquisition schemes in the intercell synchronous CDMA DL. The probabilities of correct detection and false alarm have analytically been derived, and numerical results were provided in terms of the attainable MAT performance. Ironically, our findings suggest that increasing the number of transmit antennas in CDMA system results in combining the low-energy noisecontaminated signals of the transmit antennas, which ultimately degrades the achievable MAT performance when the SINR is relatively low, regardless of whether single- or multipath scenarios are considered. This phenomenon has a detrimental effect on the attainable performance of Rake-receiver-based initial synchronization. Based on the aforementioned results justified by information theoretic considerations, our acquisition design guidelines are applicable to diverse NC MIMO-aided scenarios.

\section{REFERENCES}

[1] D. Gesbert, M. Shafi, D. S. Shiu, P. J. Smith, and A. Naguib, "From theory to practice: An overview of MIMO space-time coded wireless systems," IEEE J. Sel. Areas Commun., vol. 21, no. 3, pp. 281-302, Apr. 2003.

[2] A. J. Viterbi, CDMA: Principles of Spread Spectrum Communication. Reading, MA: Addison-Wesley, 1995, ch. 3.

[3] L.-L. Yang and L. Hanzo, "Serial acquisition of DS-CDMA signals in multipath fading mobile channels," IEEE Trans. Veh. Technol., vol. 50, no. 2, pp. 617-628, Mar. 2001.

[4] L.-L. Yang and L. Hanzo, "Differential acquisition of $m$-sequences using recursive soft sequential estimation," IEEE Trans. Wireless Commun., vol. 4, no. 1, pp. 128-136, Jan. 2005.

[5] S.-H. Won and L. Hanzo, "Non-coherent code acquisition in the multiple transmit/multiple receive antenna aided single- and multi-carrier DS-CDMA downlink," IEEE Trans. Wireless Commun., vol. 6, no. 11, pp. 3864-3869, Nov. 2007.

[6] S.-H. Won and L. Hanzo, "Analysis of serial-search-based code acquisition in the multiple-transmit/multiple-receive-antenna-aided DS-CDMA downlink," IEEE Trans. Veh. Technol., vol. 57, no. 2, pp. 1032-1039, Mar. 2008.

[7] S. Won and L. Hanzo, "Non-coherent and differentially coherent code acquisition in MIMO assisted DS-CDMA multi-path downlink scenarios," IEEE Trans. Wireless Commun., vol. 7, no. 5, pt. 1, pp. 1585-1593, May 2008.

[8] H. R. Park, "Performance analysis of a double-dwell serial search technique for cellular CDMA networks in the case of multiple pilot signals," IEEE Trans. Veh. Technol., vol. 48, no. 6, pp. 1819-1830, Nov. 1999.

[9] S. Glisic and M. D. Katz, "Modeling of the code acquisition process for rake receivers in CDMA wireless networks with multipath and transmitter diversity," IEEE J. Sel. Areas Commun., vol. 19, no. 1, pp. 21-32, Jan. 2001.

[10] S. H. Won and Y. J. Kim, "Performance analysis of multi-path searcher for mobile station in W-CDMA system employing transmit diversity," Electron. Lett., vol. 39, no. 1, pp. 137-139, Jan. 2003.

[11] S. X. Ng and L. Hanzo, "On the MIMO channel capacity of multidimensional signal sets," IEEE Trans. Veh. Technol., vol. 55, no. 2, pp. 528-536, Mar. 2006.

[12] J. G. Proakis, Digital Communications, 4th ed. New York: McGrawHill, 2001, ch. 2, pp. 17-79.

[13] G. E. Corazza, C. Caini, A. Vanelli-Coralli, and A. Polydoros, "DSCDMA code acquisition in the presence of correlated fading-Part I: Theoretical aspects," IEEE Trans. Commun., vol. 52, no. 7, pp. 11601168, Jul. 2004.

[14] G. E. Corazza, C. Caini, A. Vanelli-Coralli, and A. Polydoros, "DSCDMA code acquisition in the presence of correlated fading-Part II: Application to cellular networks," IEEE Trans. Commun., vol. 52, no. 3 , pp. 1397-1407, Mar. 2004 
[15] R. N. McDonough and A. D. Whalen, Detection of Signals in Noise, 2nd ed. New York: Academic, 1995, ch. 5.

[16] S. Kim, "Effect of spatial fading correlation on CDMA code-acquisition performance," Proc. Inst. Elect. Eng.-Commun., vol. 152, no. 1, pp. 103112, Feb. 2005.

[17] L. Hanzo, M. Münster, B. J. Choi, and T. Keller, "OFDM and MC-CDMA for broadcasting multi-user communications," in WLANs and Broadcasting. Hoboken, NJ: Wiley, 2003.

[18] V. Tarokh, A. Naguib, N. Seshadri, and A. R. Calderbank, "Space-time codes for high data rate wireless communication: Performance criteria in the presence of channel estimation errors, mobility, and multiple paths," IEEE Trans. Commun., vol. 47, no. 2, pp. 199-207, Feb. 1999.

[19] J.-C. Lin, "Differentially coherent PN code acquisition with full-period correlation in chip-synchronous DS/SS receivers," IEEE Trans. Commun., vol. 50, no. 5, pp. 698-702, May 2002.

[20] 3GPP TS 25.101 V4.11.0, User Equipment (UE) Radio Transmission and Reception (FDD).

[21] H.-C. Wang and W.-H. Sheen, "Variable dwell-time code acquisition for direct-sequence spread-spectrum systems on time-variant Rayleigh fading channels," IEEE Trans. Commun., vol. 48, no. 6, pp. 1037-1046, Jun. 2000.

[22] O. S. Shin and K. B. Lee, "Differentially coherent combining for doubledwell code acquisition in DS-CDMA systems," IEEE Trans. Commun., vol. 51, no. 7, pp. 1046-1050, Jul. 2003.

[23] W.-H. Sheen, J.-K. Tzeng, and C.-K. Tzou, "Effects of cell correlations in a matched-filter PN code acquisition for direct-sequence spread-spectrum systems," IEEE Trans. Veh. Technol., vol. 48, no. 3, pp. 724-732, May 1999.

[24] T. L. Marzetta and B. M. Hochwald, "Capacity of a mobile multipleantenna communication link in Rayleigh flat fading," IEEE Trans. Inf. Theory, vol. 45, no. 1, pp. 139-157, Jan. 1999.

[25] L. Zheng and D. N. C. Tse, "Communication on the Grassmann manifold: A geometric approach to the noncoherent multiple-antenna channel," IEEE Trans. Inf. Theory, vol. 48, no. 2, pp. 359-383, Feb. 2002.

[26] C. Rao and B. Hassibi, "Analysis of multiple-antenna wireless links at low SNR," IEEE Trans. Inf. Theory, vol. 50, no. 9, pp. 2123-2130, Sep. 2004

[27] G. J. Foschini, "Layered space-time architecture for wireless communication in a fading environment when using multi-element antennas," Bell Labs. Tech. J., vol. 1, no. 2, pp. 41-59, 1996.

[28] Y.-P. E. Wang and T. Ottosson, "Cell search in W-CDMA," IEEE J. Sel. Areas Commun., vol. 18, no. 8, pp. 1470-1482, Aug. 2000.

[29] S. Fukumoto, K. Higuchi, M. Sawahashi, and F. Adachi, "Experiments on space time block coding transmit diversity (STTD) in W-CDMA forward link," IEICE Trans. Fundam., vol. E84-A, no. 12, pp. 30453057, 2001.

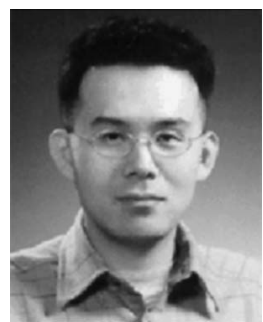

Seung Hwan Won (M'04) received the B.S. and M.S. degrees in radio science and engineering from Korea University, Seoul, Korea, in 1999 and 2001, respectively. $\mathrm{He}$ is currently working toward the Ph.D. degree with the Communications Research Group, School of Electronics and Computer Science, University of Southampton, Southampton, U.K.

From January 2001 to September 2004, he was a Research Engineer with the Mobile Communication Technology Research Laboratory, LG Electronics R\&D, Anyang, Korea. His research interests include initial synchronization in noncoherent multiple-input-multiple-output (MIMO)-aided single-carrier and multicarrier code division multiple access, interleave division multiple access, and orthogonal frequency-division multiple access, and in iterative synchronization schemes designed for MIMO-aided single-carrier and multicarrier transmission systems.

Dr. Won was the recipient of the 2004 State Scholarship of the Information and Telecommunication National Scholarship Program, Ministry of Information and Communication, Korea.

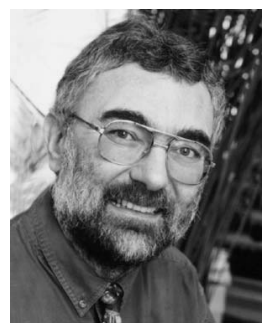

Lajos Hanzo (M'91-SM'92-F'04) received the M.S. degree in electronics and the Ph.D. degree from Technical University of Budapest, Budapest, Hungary, in 1976 and 1983, respectively, and the D.Sc. degree from the University of Southampton, Southampton, U.K., in 2004.

During his career in telecommunications, he has held various research and academic posts in Hungary, Germany, and the U.K. Since 1986, he has been with the School of Electronics and Computer Science, University of Southampton, where he holds the chair in telecommunications. He currently heads an academic research team working on a range of research projects in the field of wireless multimedia communications sponsored by industry; the Engineering and Physical Sciences Research Council, U.K.; the European Information Society Technologies Programme; and the Mobile Virtual Centre of Excellence, Basingstoke, U.K. $\mathrm{He}$ is an enthusiastic supporter of industrial-academic liaison and offers a range of industrial courses. He has coauthored 15 John Wiley and IEEE Press books totaling 10000 pages on mobile radio communications, authored about 700 research papers, organized and chaired conferences, and presented various keynote and overview lectures.

Dr. Hanzo is a Fellow of the Royal Academy of Engineering and the Institution of Electrical Engineers. He is an IEEE Distinguished Lecturer of both the Communications and the Vehicular Technology Societies and is a Governor of the IEEE Vehicular Technology Society. He is an Editor for the PRoceedings of THE IEEE. He has been the recipient of a number of distinctions. 